\title{
ARTIGO
}

do https://doi.org/10.22481/praxisedu.v16i39.6378

\section{UNA INTERVENCIÓN EN MATEMÁTICA EDUCATIVA CON FOCO EN LAS PRÁCTICAS DE LOS FORMADORES A PARTIR DE TAREAS DE GENERALIZAR Y PARTICULARIZAR}

\author{
AN INTERVENTION IN EDUCATIONAL MATHEMATICS WITH A FOCUS ON \\ TEACHER EDUCATOR PRACTICES THROUGH GENERALIZING AND \\ PARTICULARIZING TASKS
}
INTERVENÇÃO EM MATEMÁTICA EDUCACIONAL, COM FOCO NAS PRÁTICAS DOS FORMADORES, ATRAVÉS DE TAREFAS DE GENERALIZAÇÃO E PARTICULARIZAÇÃO

Victoria Mesa

Universidad de La República - Uruguay Consejo de Formación en Educación - Uruguay

\author{
Verónica Molfino \\ Universidad de La República - Uruguay \\ Consejo de Formación en Educación - Uruguay \\ Cristina Ochoviet \\ Universidad de La República - Uruguay \\ Consejo de Formación en Educación - Uruguay
}

\begin{abstract}
Resumo: Neste trabalho, relatamos os resultados de uma intervenção que aborda a necessidade de promover mudanças nas práticas dos formadores de professores, para que sejam consistentes com o que se espera que se desenvolva em suas aulas os futuros docentes. Em particular, neste caso foi proposta uma intervenção com foco nas práticas de ensino realizadas por meio de um trabalho conjunto de um instrutor com uma equipe de pesquisadores. A intervenção favoreceu o raciocínio pedagógico do formador e a realização de uma aula mediada por recursos didáticos que permitiram a produção de conhecimento em sala de aula. Proporcionamos evidências de que a experiência enriqueceu, em particular, as fases de compreensão, transformação e reflexão do modelo de ação e raciocínio pedagógico, que, no futuro, poderão influenciar a tomada de decisão no desenvolvimento de cursos de geometria em que ele é responsável.
\end{abstract}

Palavras chave: Formação de professores, design de tarefas, intervenção em matemática educacional.

Abstract: In this work we report the results of an intervention that addresses the need to promote changes in teacher educator practices, in order to be consistent with those expected to be developed in their classes by future mathematics teachers. In particular, this intervention was proposed with a focus on teaching practices and consisted in a collaborative work between a teacher educator and a team of 
researchers. The intervention favored the pedagogical reasoning of the teacher educator and the staging of a class mediated by didactic resources that allowed production of knowledge in the classroom. We provide evidence that the experience enriched, particularly, the phases of understanding, transformation and reflection of the pedagogical action and reasoning model of the teacher educator, which, in the future, could influence decision-making in the development of geometry courses in which he is responsible.

Keywords: Teacher training; Task design; Intervention in educational mathematics.

Resumen: En este trabajo reportamos los resultados de una intervención que atiende la necesidad de promover cambios en las prácticas de los formadores, para que sean consistentes con las que se espera desarrollen en sus clases los futuros docentes. En particular, en este caso se propuso una intervención con foco en las prácticas docentes llevada a cabo mediante un trabajo conjunto de un formador con un equipo de investigadoras. La intervención favoreció el razonamiento pedagógico del formador y la puesta en escena de una clase mediada por recursos didácticos que permitieron la producción de conocimiento en el aula. Brindamos evidencia de que la experiencia enriqueció, en particular, las fases de comprensión, transformación y reflexión del modelo de acción y razonamiento pedagógico del formador, las cuales, a futuro, podrían incidir en la toma de decisiones en el desarrollo de los cursos de geometría en los que él es responsable.

Palabras clave: Formación de profesores, Diseño de tareas, Intervención en matemática educativa.

\section{Introducción}

El futuro profesor de matemática, a lo largo de su proceso de formación, observa y vivencia diferentes prácticas de enseñanza de los formadores que están asociadas a distintas maneras de entender la enseñanza.

El modo en que los estudiantes de profesorado aprenden los conocimientos matemáticos y la forma en que les son enseñados inciden, positiva o negativamente, en sus prácticas futuras como docentes de aula (MELLADO, 1996; MARCELO, 1994). Los futuros profesores se apropian de lo que observan y, en consecuencia, de las metodologías de trabajo experimentadas como estudiantes, más que de las recomendaciones que puedan realizarse a nivel teórico desde el campo de la matemática educativa (ME); por ello, las prácticas de enseñanza en la formación inicial deben ser consistentes con las prácticas que se espera desarrollen en sus aulas los futuros profesores de matemática (MELLADO, 1996; OCHOVIET, 2010; SANTALÓ, 1994; TICKNOR, 2012).

Investigaciones realizadas en un instituto uruguayo de formación inicial de profesores de matemática para la enseñanza media plantean la necesidad de promover cambios en las prácticas de los formadores, para que sean consistentes con las que se espera desarrollen en sus clases los futuros docentes (DALCÍN; OCHOVIET; OLAVE, 2011; OLAVE, 2013). Y, en este 
sentido, Dalcín et al. (2011) recomiendan: "emprender proyectos de trabajo que atiendan el diseño y gestión de las clases de matemática de formación docente” (p. 95).

Este trabajo parte, entonces, de la premisa de que los futuros docentes deben vivenciar metodologías de clase que propicien la producción de conocimiento matemático en el aula y en las que el formador desempeñe un rol de facilitador y promotor del aprendizaje.

En este artículo reportamos los resultados de una intervención en matemática educativa orientada a las prácticas docentes (MESA; MOLFINO; OCHOVIET; SCORZA, 2018) que fue desarrollada en un curso de geometría del cuarto año de la carrera de profesor de matemática en Uruguay, en el mismo instituto en el que se reportaron los trabajos de Dalcín et al. (2011) y Olave (2013). En esta intervención se realizaron sesiones de discusión entre los investigadores y el formador encargado del curso, en las que se dialogó acerca de la relevancia de las tareas que se ofrecen a los estudiantes en el aula, poniendo foco en el diseño de un tipo de tareas que ponen atención en similitudes y diferencias (ZASLAVSKY, 2008) y que hemos denominado tareas de generalizar y particularizar (TGyP). Además, se reflexionó acerca del rol docente en la enseñanza de la matemática en la formación de profesores. Concretamente, nos propusimos como objetivo: implementar una intervención en ME orientada a las prácticas docentes a través del diseño de tareas de generalizar y particularizar, y analizar la incidencia de este proceso en el razonamiento pedagógico de un formador de profesores de matemática.

\section{Marco conceptual}

En este trabajo reportamos los resultados de una intervención orientada a las prácticas docentes en la que se discutieron y analizaron, en un trabajo conjunto entre un formador e investigadoras, algunas metodologías de enseñanza que promueven la producción de conocimiento matemático en el aula, así como recursos con probado potencial para el aprendizaje como son las tareas que ponen atención en similitudes y diferencias (ZASLAVSKY, 2008). Nuestro foco estuvo en los procesos que fundamentan el accionar del formador con el objetivo de analizar la incidencia de esta intervención en las decisiones que este toma para el dictado de sus clases.

Como nuestro interés se posiciona en cómo este formador razona el acto de enseñar y de qué manera se ve afectado por el proceso de intervención, adoptamos la concepción de enseñanza propuesta en Shulman (2005): la enseñanza "se inicia con un acto de razón, continúa con un proceso de razonamiento, culmina con la acción de impartir, sonsacar, hacer participar, 
o seducir, y luego es objeto de mayores reflexiones hasta que el proceso puede reiniciarse" (p. 17); y agrega que la enseñanza es "un acto de comprensión y razonamiento, de transformación y reflexión" (p. 17).

Entendemos que la intervención es una experiencia cargada de reflexiones y, en consecuencia, el formador tendrá la oportunidad de razonar asuntos relativos a la enseñanza que podrían desencadenar actos de comprensión acerca de lo que enseña y cómo lo enseña. En este proceso el formador podría identificar motivos que justifiquen decisiones que conlleven a nuevas acciones pedagógicas. Tal como Shulman (2005) lo señala: "Esta imagen de la enseñanza supone el intercambio de ideas. La idea es captada, sondeada y comprendida por un profesor que, luego, tiene que darle vueltas en la cabeza para advertir sus facetas" (p. 18). Si esa idea redunda en comprensión entonces el docente podrá avanzar hacia la toma de decisiones en sus prácticas de enseñanza. Esto es, es necesaria la comprensión para lograr la acción.

El siguiente cuadro sintetiza el modelo de razonamiento y acción pedagógicos propuesto por Shulman (2005).

\section{Comprensión}

De objetivos, estructuras de la materia, ideas dentro y fuera de la disciplina.

\section{Transformación}

Preparación: interpretación y análisis crítico de textos, estructuración y segmentación, creación de un repertorio curricular y clarificación de los objetivos.

Representación: uso a partir de un repertorio de representaciones que incluye analogías, metáforas, ejemplos, demostraciones, explicaciones, etc.

Selección: escoger a partir de un repertorio pedagógico que incluye modalidades de enseñanza, organización, manejo y ordenamiento.

Adaptación y ajuste a las características de los alumnos: considerar los conceptos, preconceptos, conceptos erróneos y dificultades, idioma, cultura y motivaciones, clase social, género, edad, capacidad, aptitud, intereses, conceptos de sí mismo y atención.

\section{Instrucción}

Manejo, presentaciones, interacciones, trabajo grupal, disciplina, humor, formulación de preguntas, y otros aspectos de la enseñanza activa, la instrucción por descubrimiento o indagación, además de las formas observables de enseñanza en la sala de clases.

\section{Evaluación}

Verificar la comprensión de los alumnos durante la enseñanza interactiva. Evaluar la comprensión de los alumnos al finalizar las lecciones o unidades. Evaluar nuestro propio desempeño, y adaptarse a las experiencias.

\section{Reflexión}

Revisar, reconstruir, representar y analizar críticamente nuestro desempeño y el de la clase, y fundamentar las explicaciones en evidencias. 


\section{Nuevas maneras de comprender}

Nueva comprensión de los objetivos, de la materia, de los alumnos, de la enseñanza y de sí mismo. Consolidación de nuevas maneras de comprender y aprender de la experiencia.

Figura 1. Modelo de razonamiento y acción pedagógicos.

Fuente: Shulman (2005, p. 20)

Como puede apreciarse, un proceso de enseñanza se inicia y culmina con un acto de comprensión. Particularmente nos interesa la fase de transformación pues la selección incluye la elección de metodologías didácticas: "el profesor debe pasar desde el acto de reformular el contenido de la materia mediante representaciones hasta concretar las representaciones en formas o métodos de enseñanza" (p. 22) que es el lugar en el que principalmente ubicamos la mirada, es decir, la manera en que la intervención planificada incide en la elección de nuevos modos de acción pedagógica en el aula de la formación docente que comiencen a distanciarse de la clase expositiva. Esto nos conduce a analizar cómo la intervención podría incidir en la transformación de las ideas centrales a ser enseñadas para adaptarlas a los objetivos y alcances del curso. En ese proceso, es necesario preparar materiales específicos, pensar en posibles formas de representar el conocimiento, su estructuración y segmentación, seleccionar métodos de enseñanza específicos, adaptar métodos y conocimiento a las características del grupo, todos estos aspectos de la planificación de la clase. Es allí donde se interviene, mediante la consideración de documentos provenientes de la investigación en ME que aportan metodologías específicas para hacer posible esta transformación.

La intervención en ME orientada a las prácticas puede incidir también en la instrucción: las investigaciones provenientes del campo brindan herramientas metodológicas sobre el tipo de tareas que se proponen y cómo se proponen, qué rol se le otorga al estudiante y cuál al docente, cuáles intervenciones conviene que realice y cuáles no.

La fase de reflexión, que supone un análisis retrospectivo del proceso de enseñanza, permite volver a la enseñanza que tuvo lugar y observar qué ideas jerarquiza el docente y cómo valora los logros a nivel del docente y de los estudiantes. La intervención puede aportar a una reflexión en torno a los objetivos del curso, en particular pensándolo como un curso que se enmarca en una carrera de profesorado de matemática: ¿qué objetivos específicos se persigue al enseñar geometría avanzada para futuros profesores? ¿Son los mismos que se perseguirían, por ejemplo, en un curso para futuros matemáticos? 


\section{Método}

Intervención en Matemática Educativa orientada a las prácticas docentes

Una intervención en ME orientada a las prácticas docentes consiste en

una propuesta de acción original y creativa, programada en el tiempo y desarrollada en forma colaborativa entre un investigador y un profesor de Matemática, desde una concepción teórica determinada, que busca contribuir o generar cambios en las prácticas docentes con el objetivo de mejorar los aprendizajes. (MESA et al., 2018, p. 184)

El investigador y el profesor trabajan en forma colaborativa para que los estudiantes logren ciertos aprendizajes. La intervención no se focaliza en el estudiante, sino en brindar herramientas metodológicas al formador para enriquecer sus prácticas de enseñanza. Dichas prácticas constituyen el foco y el objeto de la intervención.

\section{$\underline{\text { Diseño }}$}

Se planifica una intervención en ME orientada a las prácticas docentes de un formador experimentado, en la asignatura "Profundización en Geometría" del último año de la carrera de profesorado de Matemática en un instituto de formación de profesores.

El modelo de intervención está basado en las siguientes etapas:

1. Se presenta al formador el plan de trabajo, en qué consiste la intervención, cuáles son sus objetivos y cuál es el rol del investigador. Se explicitan las prácticas docentes recomendadas para la formación docente (MELLADO, 1996; OCHOVIET, 2010; SANTALÓ, 1994), así como la problemática planteada por Ticknor (2012) acerca de la necesidad de establecer conexiones explícitas entre la matemática del nivel superior que se enseña y aquella que los futuros profesores habrán de enseñar.

2. Se presentan al formador herramientas metodológicas: se caracterizan y ejemplifican las tareas de atención a similitudes y diferencias (ZASLAVSKY, 2008) y específicamente las TGyP como un tipo de ellas (POLYA, 1965, 1966; DAVIS; HERSH, 1989; MALDONADO et al., 2015).

Las TGyP, conceptualizadas en Maldonado et al. (2015), forman parte de una categoría más amplia que son las que Zaslavsky (2008) identifica como tareas de atención a similitudes y diferencias entre objetos matemáticos. Tomando como punto de partida las definiciones dadas por Polya (1965) de los constructos generalizar y particularizar en la resolución de un problema matemático, en Maldonado et al. (2015) se describen las tareas de generalizar como 
aquellas que "invitan a atender propiedades de uno o más objetos matemáticos particulares y proponer que los alumnos investiguen si conjuntos de objetos, que incluyen al particular, conservan la o las propiedades originales" (pp. 83-84). Las tareas de generalizar incluyen una gran variedad de actividades, de distintos contextos matemáticos, no entienden a la generalización solo como generalización algebraica. A su vez, en las tareas de particularizar, "partiendo de una situación o problema se recurre a un caso particular para realizar ciertas conjeturas y brindar argumentos para la misma" (p. 84). En ambos tipos de tareas se pretende que el estudiante investigue no solo si un cierto resultado es válido para el caso particular o el general, sino también si los argumentos dados son válidos al transitar de un contexto al otro.

Este tipo de tareas, además de constituir interesantes problemas matemáticos, permiten que el futuro profesor resuelva problemas que tratan un cierto saber desde distintas perspectivas. Esto le brinda herramientas para problematizar el contenido a enseñar a nivel de enseñanza media. Las TGyP favorecen el contacto con metodologías de trabajo en las que el eje central es la construcción del conocimiento matemático en el aula entendida como un espacio de indagación y aprendizaje del futuro profesor.

3. Se diseñan las TGyP desde un trabajo colaborativo entre formador e investigador. Se analiza su adecuación a los objetivos y contenidos del curso, se planifica cómo será su implementación en la clase, y se discute sobre cuáles son las metodologías de trabajo más convenientes para un mayor aprovechamiento de la propuesta. Se analiza el potencial a priori de las actividades diseñadas.

4. El formador propone a los estudiantes las tareas diseñadas. En esta instancia el investigador observa para facilitar un posterior análisis crítico del desarrollo de la clase.

5. Se realiza una reunión final entre el investigador y el formador para un análisis a posteriori de la propuesta, en relación a la potencialidad de las actividades y de la metodología de trabajo utilizada, y la posibilidad de extensión a otras instancias de clase. Se planifica una entrevista semiestructurada (MESA, 2016) con preguntas relativas a cómo el docente evalúa el desarrollo de la clase y los aspectos teóricos y metodológicos de la intervención. Se fomenta la reflexión a partir de las siguientes interrogantes, dirigidas al docente del curso, pero abordadas de manera conjunta:

¿Qué opina acerca del desarrollo de la clase (sobre el aprendizaje de los conocimientos matemáticos involucrados y sobre la metodología de trabajo utilizada)?

¿Cómo se imagina que trabajará en la siguiente clase, a partir de lo elaborado por los estudiantes en esta? ¿Cómo se retoman las producciones y se institucionaliza el saber? 
¿Qué potencialidad le encuentra al marco teórico en juego en el tipo de tareas propuestas? ¿Considera que esta misma metodología podría ser útil para el desarrollo de algún otro contenido, en este curso o en otros?

¿Qué considera que le aportó la intervención didáctica como tal?

Con estas preguntas se pretende reflexionar tanto desde los aspectos didácticos y del desarrollo metodológico de la clase, como desde los conocimientos matemáticos en juego y su vínculo con la problemática inicial evidenciada por Ticknor (2012); generar una mirada desde el rol docente en la organización e institucionalización de los saberes; reflexionar sobre el marco metodológico específico de esta intervención: las tareas de generalizar y particularizar, desde una mirada posterior a su implementación, analizar qué aportaron al aprendizaje de la matemática en estudiantes de profesorado; involucrar al formador en la búsqueda de situaciones concretas que posibiliten la extensión de la metodología implementada a otras instancias del curso o a otros cursos, y así aportar evidencia acerca de si la intervención realizada aportó insumos didácticos al formador que le sean útiles una vez finalizada la misma; y por último, reflexionar sobre la intervención en ME como tal, como instancia que busca promover el desarrollo profesional docente.

\section{Desarrollo de la intervención}

En una primera instancia, se invitó a un formador, docente del curso "Profundización en Geometría", a formar parte de una intervención en ME que consistió en diseñar y proponer a los estudiantes tareas de generalizar y particularizar, a ser implementadas con una metodología específica. El docente aceptó participar. Las etapas que se desarrollaron se describen a continuación.

Las etapas (1) y (2) se desarrollaron en una misma reunión realizada entre investigador y formador responsable del curso. Se reflexiona junto al formador sobre la importancia de las tareas que proponemos a los estudiantes y sobre el rol del docente en su implementación para un mejor aprovechamiento de estas. En dicha reunión, además, el formador nos expresa los objetivos y contenidos del curso, buscando identificar, en conjunto, en qué instancias puede ser conveniente implementar este tipo de tareas. Para profundizar los contenidos presentados y brindarle insumos desde el punto de vista metodológico se le propone al formador la lectura de: Zaslavsky (2008) y Maldonado et al. (2015). 
En la siguiente reunión se desarrolló la etapa (3). Teniendo en cuenta el conocimiento del formador de las características de los estudiantes, los contenidos del curso y su experiencia como docente de la asignatura, y a partir de las herramientas metodológicas que aportaron las investigadoras, se diseñaron dos actividades para ser propuestas en clase.

Es importante señalar que previo al desarrollo de la intervención, el formador venía pensando, junto a otro docente de la institución, en generalizaciones principalmente desde el punto de vista de los contenidos matemáticos (qué generalizar y de qué modo, qué nuevas propiedades pueden conjeturarse y cuáles son válidas), pero posteriormente a las etapas 1 y 2 , comienza a planteárselo, además, desde el punto de vista didáctico, como una tarea a proponer a sus estudiantes. Las herramientas metodológicas brindadas sirvieron de insumo para ello.

A partir del problema de la existencia del baricentro en un triángulo (problema original), se elaboran las siguientes consignas:

1) “Particularizar la noción de baricentro de un triángulo a un segmento". Formador e investigadoras acordaron que esta actividad sería propuesta en una clase previa a la observada por las investigadoras. Entendemos que este es un caso particular del problema original (estudio del baricentro de una figura), para el caso en que dos de los vértices del triángulo coinciden.

2) “Generalizar la noción de baricentro de un triángulo a un cuadrilátero", que será implementada en la clase visitada por las investigadoras. La generalización del problema original consiste en definir y construir el baricentro para polígonos de cualquier cantidad de lados, pero se plantea abordar en esta primera instancia el caso de los cuadriláteros. El caso del triángulo podría verse como un caso particular del de cuadrilátero, cuando dos vértices consecutivos coinciden.

Se acuerda proponerla con los estudiantes en una modalidad de taller, en parejas, teniendo acceso al software GeoGebra, y con tiempo suficiente para que los mismos puedan formular conjeturas y refutarlas o confirmarlas.

Se planifica que el formador en una clase previa revise con los estudiantes el concepto de baricentro en triángulos, analizando los argumentos físicos y matemáticos que explican la propiedad del baricentro. Así, los estudiantes tendrán la posibilidad de analizar nuevamente los conocimientos matemáticos involucrados, vinculados a contenidos de enseñanza media, y adquirir insumos para extender la noción de baricentro a un cuadrilátero, que constituye un conocimiento matemático avanzado.

En la etapa (4) se implementan las actividades en el ámbito de clase. El formador propone las tareas diseñadas a seis estudiantes en una clase de 135 minutos de duración, que 
trabajan en parejas, donde cada una cuenta con una computadora con el software GeoGebra, acorde a lo planificado. Las investigadoras acompañan en esta instancia para facilitar el análisis crítico posterior a la puesta en escena, pero desde un rol de observadoras.

La clase se desarrolla de la siguiente manera:

I. El formador explicita la consigna de trabajo y los estudiantes, futuros profesores, comienzan a trabajar en ello.

II. Pasados 45 minutos se realiza una puesta en común en la que el formador expone los avances realizados por los equipos y se plantean ideas en las que seguir trabajando.

III. Se destina nuevamente tiempo para el trabajo de los estudiantes en los subgrupos.

IV. Pasados otros 60 minutos se realiza una última puesta en común. Los estudiantes desarrollaron diversas estrategias de resolución, algunas de ellas previstas en el análisis a priori de la actividad y otras nuevas.

V. Al finalizar el tiempo de clase, el formador plantea sugerencias en las que seguir investigando sobre el tema, a partir de lo trabajado, con la idea de retomarse en alguna clase posterior.

La etapa (5) se llevó a cabo mediante una entrevista posterior a la clase, días después, guiada por las preguntas planteadas.

\section{Observaciones y análisis de los resultados de la intervención}

Desde el inicio de la intervención, el formador se muestra interesado en participar en esta propuesta de trabajo colaborativo.

En la etapa (2) se observa en el formador un interés por abordar resultados no conocidos por los estudiantes acerca de la generalización de propiedades conocidas a nuevos contextos, principalmente resultados recientemente conocidos por la comunidad matemática, para que los estudiantes "vean a la Matemática como algo vivo, en erupción, no como algo muerto", en palabras del formador.

Previo a la intervención, el formador venía reflexionando acerca de posibles generalizaciones de conceptos geométricos, específicamente en torno a la idea física del concepto de baricentro, pero principalmente en el plano de lo matemático; no tenían aún un plan de cómo llevarlo a cabo en la clase. La intervención le proporcionó herramientas metodológicas específicas para volcar este conocimiento matemático en una tarea de aula para proponer a los estudiantes de profesorado. Entendemos que esto abonó, en particular, a la fase 
de selección del modelo de razonamiento y acción pedagógicos (SHULMAN, 2005), favoreciendo que ponga en práctica con sus estudiantes una metodología de trabajo que no ha experimentado previamente, así como la fase de representación, en lo que hace al diseño de un tipo particular de tarea para proponer a sus estudiantes.

En la etapa (4) se desarrolló una propuesta de trabajo basada en una dinámica de taller, en la que los estudiantes propusieron ideas a partir de la consigna inicial y con mínimas intervenciones del formador. El formador accedió sin dificultades a proponer esta forma de trabajo según lo acordado en la etapa (3) de la intervención, lo que entendemos incidió en la etapa de instrucción del modelo de razonamiento y acción pedagógicos. El rol del formador del curso fue principalmente de guía al momento del trabajo en los subgrupos y de expositor de lo producido en los equipos al momento de la puesta en común, además de institucionalizar ciertos saberes y aportar preguntas en las que seguir trabajando. En una primera instancia es el formador quien expone las conclusiones arribadas en los equipos, pero en una segunda instancia, a sugerencia de una de las investigadoras, son los estudiantes los que exponen sus razonamientos y observaciones. El tiempo otorgado a las producciones de los estudiantes fue adecuado, considerando los tiempos de clase.

En la clase que el formador dicta, presenta la consigna y, tal como fue acordado, da tiempo a los estudiantes para que la aborden trabajando en pequeños grupos, con disponibilidad de acceso a computadoras con el programa GeoGebra instalado. La primera idea que surge de los estudiantes para generalizar el concepto de baricentro a un cuadrilátero es construir el centro del paralelogramo de Varignon. Esta construcción permite obtener una de las generalizaciones de baricentro planificada en la etapa (3), pero la manera en que se construye es distinta a la pensada previamente. La construcción realizada por los estudiantes es una idea inmediata que concuerda con la idea física del baricentro como centro de masa considerando masas equidistribuidas en los vértices del cuadrilátero.

Posteriormente surgen ideas para una segunda construcción (de la misma generalización), que sí fue considerada en la planificación de la clase, y que consiste en definir el baricentro del cuadrilátero como el baricentro del segmento determinado por un vértice del cuadrilátero y el baricentro del triángulo determinado por sus otros tres vértices. La interrogante planteada es si ambas construcciones conducen al mismo punto y por qué.

No fue propuesta por los estudiantes otra de las generalizaciones que fueron consideradas a priori en la planificación de la clase: considerar el baricentro del cuadrilátero como el centro del cuadrilátero determinado por los baricentros correspondientes a los cuatros 
triángulos determinados por tres vértices del cuadrilátero original, a partir de la idea física de pensar el cuadrilátero con masa homogénea en toda su superficie.

Los estudiantes, con la guía del docente, buscaron argumentos para justificar sus afirmaciones desde el punto de vista matemático. Vincularon y pusieron en juego conocimientos de paralelismo, homotecia, semejanza, baricentro en triángulos, entre otros.

La etapa (5) de la intervención se llevó a cabo a partir de las preguntas planificadas, presentadas en la sección anterior. En la entrevista el formador manifiesta valorar positivamente la dinámica de clase, y considera favorable que haya surgido de los estudiantes respuestas a las consignas y que se mostraran interesados. Por otra parte, el formador sostiene que para que los estudiantes puedan demostrar, además de conjeturar, necesitan tener una base teórica que, según deja entrever, se lograría a través de clases más tradicionales. Sí considera viable la construcción de conocimientos por parte de los estudiantes en una etapa posterior. Como respuesta a la pregunta de continuidad de la metodología, menciona que tiene pensado abordar la generalización del concepto de ortocentro y la generalización de la recta de Euler, percibiendo como favorable implementar una dinámica de clase muy similar a la realizada. Apuesta a que con el tiempo surjan otras ideas nuevas y vaya adquiriendo, como parte de un proceso gradual, otra dinámica de trabajo; por ejemplo, menciona que el uso de computadoras para investigar en el ámbito de clase hasta el momento había sido poco frecuente. En este sentido el formador afirma: "yo lo pienso como una idea progresiva de ir incorporando cierto [...] tipo de ideas para poder generar actividades de este tipo y hacerlo más fluido".

Respecto a la idea de generalizar y particularizar, afirma que la misma siempre aparece en las clases de matemática de los distintos cursos, incluso en las clases más expositivas, ya que el docente puede mostrar y los estudiantes pueden observar distintas generalizaciones. Pero proponer como consigna una actividad de generalizar y particularizar a los estudiantes, es algo distinto. El formador concibe que esto es factible principalmente en cursos de geometría, no visualizando inmediatamente cómo hacerlo en otros cursos, pero no descartando la posibilidad.

En la entrevista el formador también deja entrever que necesita proponer clases tradicionales, donde desarrolle contenidos teóricos, previamente a plantear a los estudiantes clases más constructivas, ya que considera que esto es necesario para que los estudiantes tengan la posibilidad de demostrar, y no solo de observar. El formador concibe que hay contenidos que no pueden ser construidos por los estudiantes debido, por ejemplo, a que cuestiones que supone conocidas por ellos de cursos anteriores no las recuerdan. 
Por otra parte, sostiene que ambos tipos de clases, entiéndase expositivas y constructivas, deberían coexistir en el curso y lo expresa de la siguiente manera:

(...) todo es cuestión de buscar un equilibrio, de ir introduciendo este tipo de cosas, ir haciendo clases un poco más tradicionales y logrando avanzar en que ellos estudien ciertos contenidos, y bueno después ir apostando a que descubran algunas propiedades interesantes, para mí es tema de irlo mechando.

Cuando se lo interroga acerca del uso de las computadoras en la modalidad de taller en el aula y nos cuenta sobre la poca frecuencia con que lo hace, agrega: "soy de los convencidos de que los cambios se van haciendo de a poco". A partir de esta mención observamos el posicionamiento del formador (si bien lo dice en forma impersonal) frente a un cambio, mostrando en cierto sentido un interés por cambiar sus prácticas.

Respecto a proponer este marco metodológico en otros contenidos y en otros cursos, el formador percibe el potencial en geometría y se anima en esta área.

El formador aprecia como una experiencia positiva el haber trabajado en una modalidad de taller, de manera que los estudiantes puedan descubrir cosas nuevas por su cuenta y confiar en su capacidad para demostrarlas. Manifiesta que tiene pensado en una futura clase "trabajar con generalizar el concepto de ortocentro y, en definitiva, terminar generalizando el concepto de recta de Euler. [....] Que creo que también con un trabajo muy similar puede salir muy lindo", refiriéndose a la propuesta de taller y al trabajo de los estudiantes con computadora. En la entrevista el formador explica cómo planifica este trabajo futuro, pensando concretamente en cuál será el desarrollo del trabajo en el aula.

La siguiente cita da la pauta de que la intervención fue exitosa en sus objetivos, dado que el acercarle al formador herramientas metodológicas concretas, lo anima a plantear este tipo de actividades en la clase, viendo esto como un camino posible para modificar sus prácticas, aunque no lo manifieste de manera explícita:

Cuando empecé tenía en la cabeza una idea, ahora a partir de esto ya tengo dos más. Capaz que de aquí a un par de años, son seis, siete u ocho, bueno y eso te da otra dinámica, ¿no? Además, en la medida que vos lo lográs pensar y lo vas aceitando podés hacerlo con una economía de tiempo también.

El formador concibe la posibilidad de incorporar a sus clases de forma progresiva nuevas metodologías de trabajo. Valoramos positivamente que considere que otras formas de abordar algunos conocimientos sean posibles y que pueda percibirse como el generador de su propio cambio. Esto hace patente un acto de comprensión, en particular cuando el formador 
dice "lo lográs pensar", y deja en evidencia que tal como lo afirma Shulman (2005), es necesario comprender para enseñar.

Consideramos que, si bien el conocimiento matemático en juego en la propuesta ya era previamente conocido por él, la intervención le acercó la metodología con la cual llevarlo a cabo. Así lo expresa el formador:

el material de baricentro lo conozco hace mucho tiempo, esta idea de generalizar el baricentro, también conocía la de generalizar el ortocentro y el circuncentro, pero no conocía determinados materiales que me permitieron verlo de otra forma. O sea, había argumentos que yo no tenía en la cabeza, conocía por otro lado ciertas pruebas, pero bueno, te abre la cabeza y te permite organizar mejor el conocimiento y por lo tanto plantearlo mejor, eso sin duda. Me genera muchas posibilidades de decir bueno: hay material para seguir trabajando sobre esto. Yo lo pienso como una idea progresiva de ir incorporando cierto [...] tipo de ideas para poder generar actividades de este tipo y hacerlo más fluido.

El formador deja entrever aquí también procesos característicos de la fase de transformación (Shulman, 2005), dando cuenta en forma explícita de que ahora posee argumentos para seleccionar contenidos, maneras de presentarlos a los estudiantes y apertura hacia la implementación de nuevas dinámicas de aula, todo esto a partir de una instancia específica de reflexión, originada a partir de la experiencia y la entrevista posterior.

\section{Discusión}

El intercambio de ideas con el formador, en las primeras etapas de la intervención, sobre el rol docente recomendado para desarrollar en clase tareas de particularizar y generalizar contribuyó al desarrollo de una dinámica que permitió la producción matemática por parte de los estudiantes, ya que el formador incorporó a su clase ciertos aspectos de dicha propuesta.

Respecto a los aportes de la intervención, el formador menciona que esta le permitió hacerse el espacio para llevar al aula una propuesta con estas características. Además, si bien el conocimiento matemático en juego ya era previamente conocido por él, le permitió conocer determinados enfoques didácticos que le dieron la posibilidad de mirar ese conocimiento desde otro lugar, logrando organizarlo y plantearlo de manera diferente, dando cuenta de los aportes de la intervención a la fase de transformación (SHULMAN, 2005). 
El formador también muestra interés por incorporar a sus clases nuevas metodologías que enfaticen en la producción de conocimientos por parte de los estudiantes, aunque esto sea realizado en forma paulatina.

Las características de la entrevista semiestructurada que fue planteada como una instancia de intercambio de ideas con el formador permitió avanzar hacia la fase de reflexión (SHULMAN, 2005) pues el formador hizo explícitas sus apreciaciones acerca de lo sucedido en clase, tanto a nivel personal como de los estudiantes y esbozando a mediano y largo plazo, la posibilidad de continuar trabajando en la incorporación de recursos que faciliten su rol de mediador en el aula de la formación de profesores de matemática.

\section{Reflexiones finales}

En este trabajo reportamos los resultados de una intervención que atiende recomendaciones específicas de la comunidad de investigadores, relativas a la necesidad de implementar proyectos que atiendan al diseño y gestión de las clases de matemática de formación docente. En particular, en este caso se propuso una intervención, proyecto con características particulares, por tratarse de un trabajo conjunto de un formador con un equipo de investigadoras.

El proceso llevado a cabo dio cuenta de la pertinencia de la intervención para fortalecer el conocimiento didáctico del contenido del formador. Él aportó su conocimiento de la materia, relativo a cómo generalizar una noción abordada en su curso y también en cursos de enseñanza básica, y también su conocimiento curricular y de los estudiantes. Ahora, las herramientas metodológicas brindadas por las investigadoras y el trabajo colaborativo con estas le dieron insumos para proponer a sus estudiantes una secuencia de tareas que permitiera abordar la generalización que él ya había considerado previamente.

La intervención favoreció, fundamentalmente, las fases de transformación y reflexión del modelo de razonamiento y acción pedagógicas (SHULMAN, 2005) pues el trabajo conjunto entre investigadoras y formador, y la puesta en escena de una clase mediada por recursos didácticos que permitieron la producción de conocimiento en el aula, favoreció procesos de razonamiento y actos de comprensión del formador, que a futuro, podrían incidir en la toma de decisiones relativos al desarrollo de los cursos de geometría del formador participante. 


\section{REFERENCIAS}

DALCÍN, Mario; OCHOVIET, Cristina; OLAVE, Mónica. Una mirada a las prácticas de los formadores de los futuros profesores de matemática: el profesor, el conocimiento y la enseñanza. Unión. Revista Iberoamericana de Educación Matemática, 28, p. 85-97, dic. 2011.

DAVIS, Philip; HERSH, Reuben. Experiencia matemática. Sección: Generalización. p. 105107. Barcelona: MEC y Labor, 1989.

MALDONADO, Ana; MEDINA, Leticia; MESA, Victoria; MOLFINO, Verónica; OCHOVIET, Cristina; PAGÉS, Daniela; RIVERO, Florencia. Tareas enfocadas a similitudes y diferencias como motor para el aprendizaje de la matemática: nuevas categorías. En Buendía, G., Molfino, V., Ochoviet, C. Estrechando lazos entre investigación y formación en Matemática Educativa. Vol. II. Consejo de Formación en Educación. Departamento de Matemática. Uruguay, 2015.

MARCELO, Carlos. Investigaciones sobre prácticas en los últimos años: qué nos aportan para la mejora cualitativa de las prácticas. Ponencia presentada al III Symposium Internacional sobre Prácticas Escolares, Poio, 1994.

MELLADO, Vicent. Concepciones y prácticas de aula de profesores de ciencias, en formación inicial de primaria y secundaria. En Investigación y experiencias didácticas. Enseñanza de las ciencias, 14 (3), 289-302, 1996.

MESA, Victoria. Actividades de generalizar y particularizar como medio para vincular la Matemática avanzada y la Matemática escolar en la formación de profesores de Matemática (tesina de Diploma no publicada). Consejo de Formación en Educación Universidad de la República. Montevideo, Uruguay, 2016.

MESA, Victoria; MOLFINO, Verónica; OCHOVIET, Cristina; SCORZA, Verónica. Intervención en Matemática Educativa orientada a las prácticas docentes: un aporte a su conceptualización y diseño. Revista Docência do Ensino Superior, v. 8, n. 2, 178-194, 2018. Disponible em https://periodicos.ufmg.br/index.php/rdes/article/view/2497

OCHOVIET, Cristina. ¿Quiénes serán los futuros formadores? Actas del II Congreso Nacional e Internacional de Formación Docente, 41-45. Montevideo: ANEP-CFE, 2010.

OLAVE, Mónica. Modelos de profesores formadores de Profesores de Matemática: ¿cuáles son y en qué medida se transmiten a los futuros docentes? Un estudio de casos. Tesis doctoral no publicada. CICATA, IPN. México, 2013. Recuperado el 1 de octubre de 2015 desde http://www.matedu.cicata.ipn.mx/tesis/doctorado/olave_2013.pdf

POLYA, George. Cómo plantear y resolver problemas. Ed. Trillas. México, 1965.

POLYA, George. Matemáticas y razonamiento pausible. Madrid: Tecnos, 1966.

SANTALÓ, Luis y colaboradores. Enfoques. Hacia una didáctica humanista de la matemática. Buenos Aires: Troquel Educación, 1994. 
SHULMAN, Lee. Conocimiento y enseñanza: Fundamentos de la nueva reforma. Knowledge and Teaching: Foundations of the New Reform. Profesorado. Revista de currículum y formación del profesorado, 9, 2. p. 1-30, 2005.

TICKNOR, Cindy. Situated learning in an abstract algebra classroom. Educ Stud Math 81: 307-323, 2012.

ZASLAVSKY, Orit. Attention to similarities and differences: A fundamental principle for task design and implementation in mathematics education. Invited presentation at the Topic Study Group (TSG34) on Research and Development on Task Design and Analysis, the 11th International Congress on Mathematics Education (ICME-11), Monterrey, México, 2008. Recuperado el 10 de octubre de 2015 desde http://tsg.icme11.org/document/get/290

\section{SOBRE AS AUTORAS:}

\section{Victoria Mesa}

Diploma en Matemática, mención Enseñanza (CFE - UdelaR, Uruguay), Profesora de Matemática de Enseñanza Media (IPA, Uruguay); Profesora en el Consejo de Educación Secundaria, Uruguay. E-mail: vickymesa_16@hotmail.com

(iD http://orcid.org/0000-0003-3166-9025

\section{Verónica Molfino}

Doctora en Matemática Educativa (CICATA, IPN, México), Profesora de Matemática de Enseñanza Media (IPA, Uruguay); Profesora e investigadora en Consejo de Formación en Educación, Uruguay; Integrante de Comité Académico del Diploma en Matemática (CFE UdelaR, Uruguay); Equipo de investigación "Identidad y conocimiento del profesor". E-mail: veromolfino@gmail.com

(iD) http://orcid.org/0000-0002-6672-762X

\section{Cristina Ochoviet}

Postdoctorado en Ciencias Sociales (UNC, CEA, Argentina). Dra. en Matemática Educativa (CICATA, IPN, México), Profesora de Matemática de Enseñanza Media (IPA, Uruguay); Profesora e investigadora en Consejo de Formación en Educación, en la Universidad de La República (UdelaR), Uruguay; Integrante de Comité Académico del Diploma en Matemática (CFE - UdelaR, Uruguay); Equipo de investigación "Identidad y conocimiento del profesor". E-mail: cristinaochoviet@gmail.com

(iD http://orcid.org/0000-0001-9069-3469 